\title{
Correction to: Transcriptome analyses of tumor-adjacent somatic tissues reveal genes co-expressed with transposable elements
}

Nicky Chung ${ }^{1 \dagger}$, G. M. Jonaid ${ }^{1 \dagger}$, Sophia Quinton ${ }^{1 \dagger}$, Austin Ross $^{1 \dagger}$, Corinne E. Sexton ${ }^{1}$, Adrian Alberto ${ }^{2}$, Cody Clymer ${ }^{2}$, Daphnie Churchill², Omar Navarro Leija ${ }^{2}$ and Mira V. Han ${ }^{1,3^{*}}$

\section{Correction to: Mobile DNA}

https://doi.org/10.1186/s13100-019-0180-5

Following publication of the original article [1], the authors reported errors in Table 2 wherein all "KZFP" in the gene names should be changed to "ZNF".

The correct version of the table is provided here.

\section{Author details}

'School of Life Sciences, University of Nevada, Las Vegas, NV 89154, USA. 2Department of Computer Science, University of Nevada, Las Vegas, NV

89154, USA. ${ }^{3}$ Nevada Institute of Personalized Medicine, Las Vegas, NV 89154, USA.

Published online: 10 October 2019

\section{Reference}

1. Chung N, et al. Transcriptome analyses of tumor-adjacent somatic tissues reveal genes co-expressed with transposable elements. Mobile DNA. 2019; 10:39 https://doi.org/10.1186/s13100-019-0180-5.

\footnotetext{
*Correspondence: mira.han@unlv.edu

${ }^{+}$Nicky Chung, G. M. Jonaid, Sophia Quinton and Austin Ross contributed equally to this work.

${ }^{1}$ School of Life Sciences, University of Nevada, Las Vegas, NV 89154, USA

${ }^{3}$ Nevada Institute of Personalized Medicine, Las Vegas, NV 89154, USA

Full list of author information is available at the end of the article
} 
Table 2 KZFP gene members in core TE modules. a. KZFP genes that are members of the core TE modules. b. KZFP genes in module M3. M3 is in high correlation with the core TE modules (Supplementary Figure 5 a. and b.)

\begin{tabular}{|c|c|c|}
\hline core TE modules & KZFP & chromosome \\
\hline \multirow[t]{5}{*}{ M8 } & HKR1 & 19 \\
\hline & ZNF226 & 19 \\
\hline & ZNF682 & 19 \\
\hline & ZNF789 & 7 \\
\hline & ZNF814 & 19 \\
\hline \multirow[t]{4}{*}{ M21 } & ZNF404 & 19 \\
\hline & ZNF418 & 19 \\
\hline & ZNF589 & 3 \\
\hline & ZNF75A & 19 \\
\hline M38 & ZNF117 & 7 \\
\hline \multirow[t]{5}{*}{ M45 } & ZNF334 & 20 \\
\hline & ZNF493 & 19 \\
\hline & ZNF506 & 19 \\
\hline & ZNF721 & 4 \\
\hline & ZNF737 & 19 \\
\hline \multicolumn{3}{|l|}{ b. } \\
\hline KZFPs in module M3 & \multicolumn{2}{|l|}{ chromosome } \\
\hline ZNF169 & \multicolumn{2}{|l|}{9} \\
\hline ZNF202 & \multicolumn{2}{|l|}{11} \\
\hline ZNF266 & \multicolumn{2}{|l|}{19} \\
\hline ZNF300 & \multicolumn{2}{|l|}{5} \\
\hline ZNF320 & \multicolumn{2}{|l|}{19} \\
\hline ZNF431 & \multicolumn{2}{|l|}{19} \\
\hline ZNF439 & \multicolumn{2}{|l|}{19} \\
\hline ZNF44 & \multicolumn{2}{|l|}{19} \\
\hline ZNF587 & \multicolumn{2}{|l|}{19} \\
\hline ZNF662 & \multicolumn{2}{|l|}{3} \\
\hline ZNF7 & \multicolumn{2}{|l|}{8} \\
\hline ZNF700 & \multicolumn{2}{|l|}{19} \\
\hline ZNF708 & \multicolumn{2}{|l|}{19} \\
\hline ZNF714 & \multicolumn{2}{|l|}{19} \\
\hline ZNF732 & \multicolumn{2}{|l|}{4} \\
\hline ZNF83 & \multicolumn{2}{|l|}{19} \\
\hline ZNF841 & \multicolumn{2}{|l|}{19} \\
\hline
\end{tabular}

